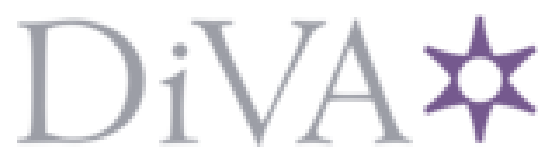

http://www.diva-portal.org

This is the published version of a paper presented at ASME 2004 International Mechanical Engineering Congress and Exposition (IMECE2004).

Citation for the original published paper:

Sandberg, S., Kalhori, V., Larsson, T. (2004)

FEM simulation supported knowledge enabled engineering in high strength steel car body design.

In: (pp. 907-913). Anaheim, USA

http://dx.doi.org/10.1115/IMECE2004-61584

N.B. When citing this work, cite the original published paper.

Permanent link to this version:

http://urn.kb.se/resolve?urn=urn:nbn:se:bth-11303 
IMECE2004-61584

\section{FEM SIMULATION SUPPORTED KEE IN HIGH STRENGTH STEEL CAR BODY DESIGN}

\author{
Stefan Sandberg \\ Division of Computer Aided Design \\ Luleå University of Technology \\ SE-971 87 Luleå, Sweden \\ Stefan.Sandberg@Itu.se
}

\author{
Vahid Kalhori \\ Division of Computer Aided Design \\ Luleå University of Technology \\ SE-971 87 Luleå, Sweden \\ Vahid.Kalhori@Itu.se
}

\author{
Tobias C. Larsson \\ Division of Computer Aided Design \\ Luleå University of Technology \\ SE-971 87 Luleå, Sweden \\ Tobias.C.Larsson@Itu.se
}

\begin{abstract}
With an increasing number of and also more complex demands on today's automobiles the need for fast and accurate simulations to support the Engineering Design (ED) process is getting more important. The demands that are put on the automotive designs are often contradictory i.e. weight against stiffness, and no one optimal set of solutions can be found, rather a trade-off situation. At Volvo Cars Corporation, known all over the world for their safety policy, the advancement to more high strength materials is causing new problems for the engineers. As widely known, a steel material that has been exposed to plastic deformation will suffer hardening in those areas. The work in this paper, exemplified in a deployed demonstrator, show that it is possible to combine forming and crashworthiness simulations in an automated way to make advanced simulation accessible to more people in the product development process. The Knowledge Enabled Engineering (KEE) demonstrator combines forming and crashworthiness simulations for dealing with the constant trade-off in ED.
\end{abstract}

\section{INTRODUCTION}

To stay competitive in a global market push Volvo Car Corporation (VCC) to make its product development process more efficient. The recent trends in automotive body structure development in conjunction with tougher demands on less environmental influence, better crashworthiness and shorter product lead-time give rise to make the product development process more efficient.

The most critical phase of the product development process is obviously the preliminary design stage [1]. At this phase there exists most opportunities to make changes in the design. In the subsequent phase of detailed design, it becomes extremely difficult, or even impossible to compensate or to correct the shortcomings of a poor design concept formulated at the conceptual design phase. Decisions made during this step influence greatly the final cost, performance and reliability of a product, Figure 1. It is therefore important to account for downstream effects in the conceptual phase. Downstream knowledge should be captured and made available in the early stages in order to lower the level of abstraction before any decisions are made, Figure 2. However, comparing product generations often reveal a large level of similarity. 


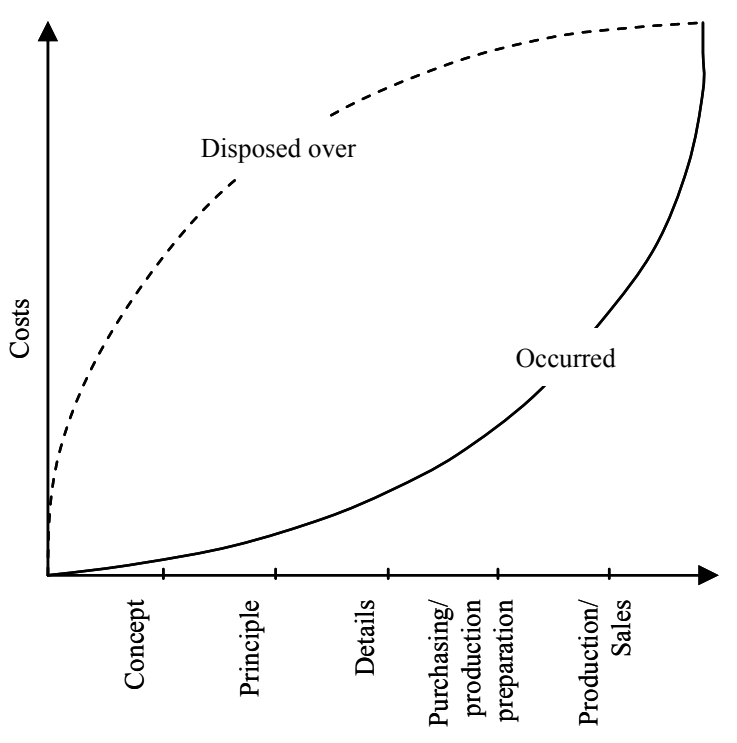

Figure 1. A large part of the costs are allocated in the early stages of the PD process, [2]

It has been argued [2] that this knowledge can be used to define engineering design [3] systems support that allows a more detailed conceptual study which in theory would reduce the level of abstraction. This means that the companies possess knowledge that can be used to further develop their products or service concepts. Traditionally experiences are transferred in design teams by members from different domains. However, there might still be knowledge gaps due to the product complexities. It is possible to include this knowledge into product models. Generative modeling has proven to support such task [4].

Efforts in combining Finite Element Methods with KEE has been made by Pinfold \& Chapman [5] and Isaksson [2] where Pinfold et al. propose a rule base method concerning the creation of first geometry of the vehicle structure and then from this a simplified model for mesh generation and then finally the generation of the $\mathrm{FE}$ mesh itself. Isaksson [2] proposes a similar approach and emphasizes the opportunity given to study a wider set of design variations than what is traditionally possible using most parametric strategies.

The objective of this study is to show how the design cycle for the car body at VCC could be shorten utilizing the knowledge enable engineering, KEE, supported by finite element simulation tools. These tools will enable the designers with limited knowledge about FE-simulation to incorporate existing designs as a starting point and build on them to arrive at updated design variations. The users can add just the contemplated modifications, and rerun the analysis and simulation to see what the planned changes will bring.
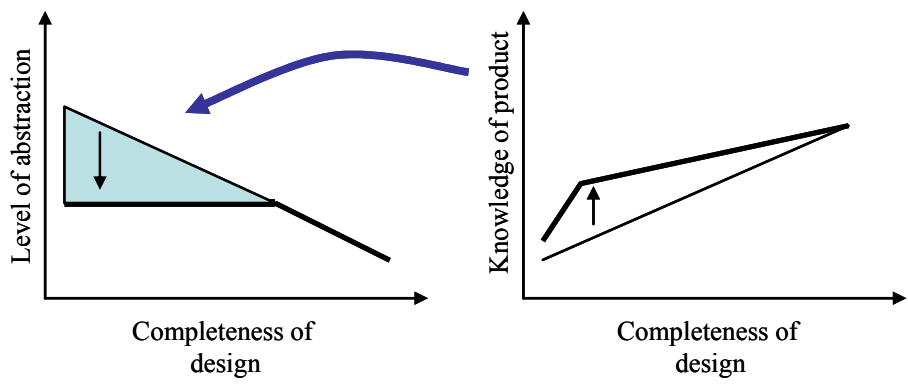

Figure 2. In the beginning of the development process the level of abstraction is high. By increasing the level of knowledge the level of abstraction is reduced allowing better decisions [2]

\section{FINITE ELEMENT SIMULATIONS}

Finite element simulations have been extensively used as an integral part in the design process to provide a deeper understanding concerning the performance of design in service and good support during the iterative procedure of synthesisanalysis loops. Design validation/verification strategies that combine simulation techniques can be effective in ensuring correct operation of software and hardware systems.

The use of experiments concerning product performance for linear stress analysis has been reduced to less than $1 \%$ compared to the pre-FE time [6]. However, the industrial use of FEM in simulation of the manufacturing process is less common and varying depending on what manufacturing process it is, Figure 3. The accuracy of finite element simulation models of different manufacturing processes is highly dependent on the modeling of the thermal and mechanical boundary conditions and inelastic material properties correct. The latter has some additional complications compared with normal nonlinear deformation problems. The manufacturing process is usually also associated with micro structural changes in the material further complicating the modeling of the material behavior.
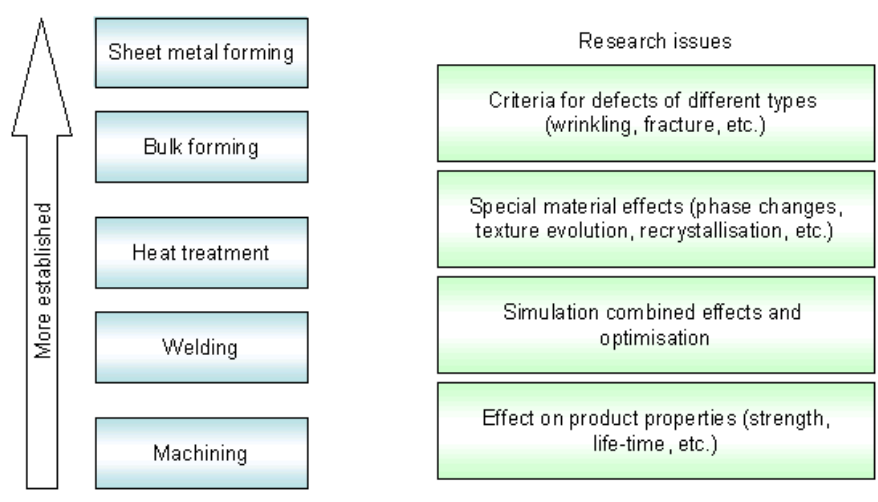
As it appears in Figure 3, finite element simulation tools are quite immature for prediction of behavior of the machining process [7]. This is due to the involved complex phenomena and their requirements on the numerical algorithms to model the extremely large deformations, the plastic flow and heat generation that are important for the cutting mechanics [8]. These models may predict cutting forces fairly well [9] but not concerning residual stresses that are important for life-cycle determination.

The use of FEM in sheet metal forming is quite established and there are conferences devoted completely to this subject. These kinds of simulations are used on a daily basis at VCC. However, there are still problems with prediction of springback as well as research issues concerning prediction of surface defects, wrinkling, tool wear etc $[10,11]$. Solid forming processes have a similar status as sheet metal forming, maybe somewhat less established due to problems of large deformations and also the fact that there are fewer large companies with competence to perform the simulations. Then there is a set of processes like heat treatment [12], welding [13, 14] that are less common to simulate in industry due to more difficult modeling and/or numerical problems. Casting simulations are also performed for very complex geometry like engine blocks but the complexity is at the level of heat treatment and welding processes.

The simulation of a chain of manufacturing processes is naturally much less common than the study of individual processes. There has been some work in combining welding and heat treatment simulations [15], even combined with CFD simulations of fluid flow in oven [16]. A three-step process was studied in [17]. There is also very little done in integrating the results from manufacturing simulations with the stresses due to the in-service loads for prediction of performance and/or life time [18]. For example, welding and fatigue [19] is based on full-scale testing and there is no documentation about welding residual stresses for these cases.

In the present study the effect of previous manufacturing process, i.e., sheet metal forming of high strength steel on crashworthiness of car body is of special interest. However, the major emphasis is on enabling the simulation of manufacturing and functional performance utilizing the existing FE-tools. This involves the development and evaluation of a tool for interactive, user-friendly manipulation of models and mapping between models. Furthermore, the use of Knowledge Enable Engineering (KEE) [2, 20] will make advanced simulations available for a designer thus speeding up the design process. The simulation experts will then embody their competence in this tool enabling the designer to do advanced simulation directly during the design process.

\section{SIMULATION SUPPORT FOR CAR DESIGN AT VCC}

At the car body department at VCC a break-down strategy to a property based model (PBM) has been developed, the car body is divided into sub-elements, beams and joints for which broken down requirements exists [21], Figure 4-5. Furthermore easy-to-use software has been developed to permit designer with less analysis experiences perform preliminary analysis on these sub-elements. The software called DAMIDA is used to transfer the CAD data, generate mesh and boundary condition to the explicit finite element code RADIOSS for crashworthiness analysis of beams.

With these tools the designer is able to get a result, showing the stiffness, plastic bending capacity and axial plastic crush capacity of the beam hence the amount of energy it would absorb in a crash. The simulation can be performed within a couple of hours. Without these tools a design engineer has to wait 4-6 weeks to get results from the complete body simulation performed by the analysis department [21].

These preliminary simulations do not replace the later simulation on complete body level, but is a mean for the design engineer to check his design against broken down requirements, as well as for fast relative comparison between different solution concepts, see Figure 5. A more precise simulation will be made later by a senior analyst before the product hits the market.

DAMIDA is though limited to only simulating the crashworthiness of the modeled beam and doesn't account for the process to form the sheet into the beams form. As widely known, high strength steel that has been exposed to plastic deformation will suffer considerable work hardening in those areas. Therefore, it is necessary to further develop the tool in order to account for the influences of earlier stage of manufacturing process on crashworthiness. This will provide he designers a more realistic design evaluation tool. The next chapter is describing further development of the presented tools for crashworthiness evaluation. It is shown the possibility for designer without extensive knowledge in simulation to evaluate and to get a quick and relatively precise answer their ideas utilizing a sequential forming and crashworthiness simulations in an automated way. 


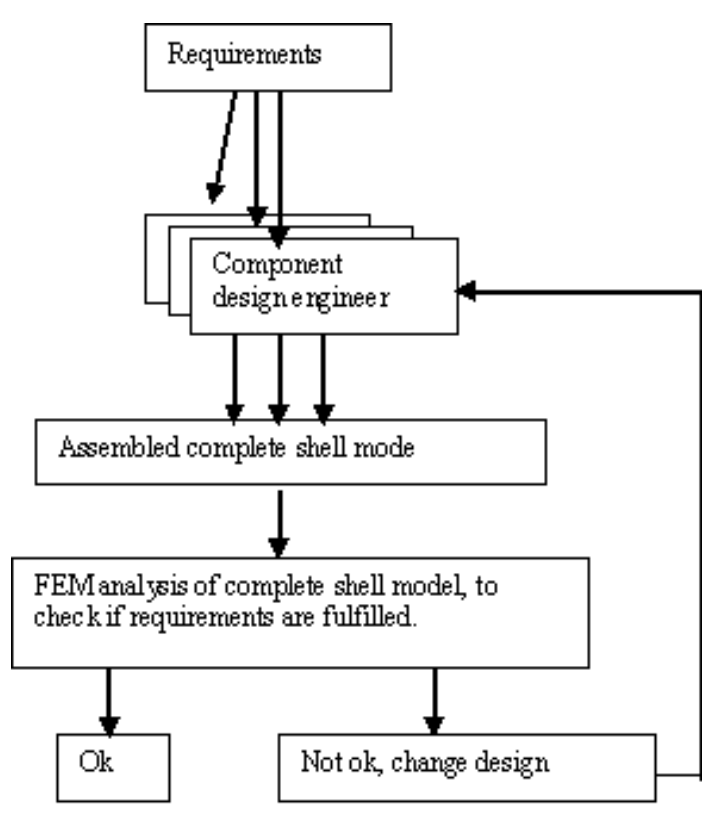

Figure 4. The traditional process for car body design with respect to mechanical properties. [20]

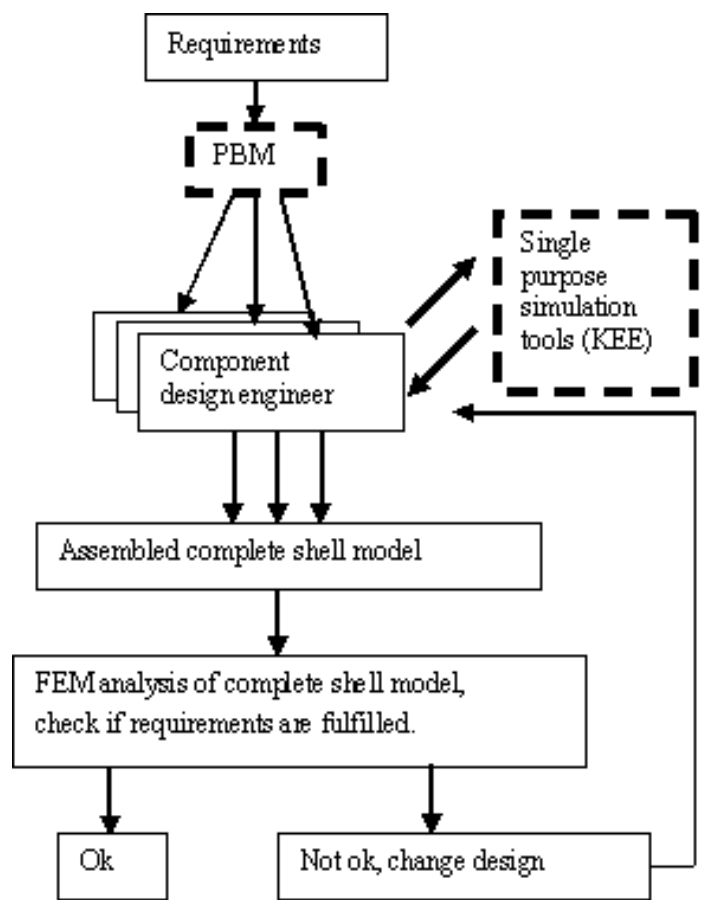

Figure 5. Change of flow with use of KEE application which allows the designer time to investigate more different solutions to meet the requirements. Time between design and analysis result is between 3-12 hours depending on complexity of the geometry being analyzed. [20]

\section{THE KEE DEMONSTRATOR}

The structure and function of what we call it the KEE demonstrator is described in details in this chapter.

\subsection{Overview of demonstrator}

During the conceptual phase of a design process it occur a number of concept generation and concept evaluation loops. This iterative process requires a number of analysis stages to evaluate the synthesis part of design process. In other word, this delay could disturb the dynamic and creative concept generation process. The idea is to let the design engineers work in the three dimensional CAD environment as they use to do and testing different concepts for, e. g., beams. The suggested tool will enable them to evaluate these ideas.

The analysis step will begin by the export of a cross section of beam as an IGES-file. The choice of using cross sections rather than the actual geometry of the beam is naturally a simplification of the problem but it is shown [21] that this is a reasonable strategy.

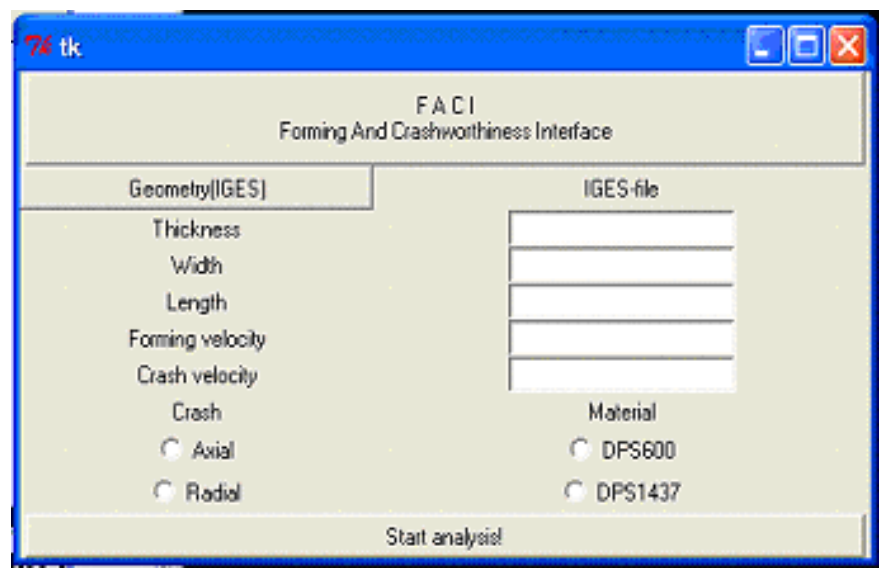

Figure 6. The graphical interface. As it can be seen only the most crucial parameters can be altered and the rest is automatic.

It gives the designer knowledge about the stiffness, plastic bending capacity and axial plastic crush capacity at various points on the beam. The cross section is then extruded in normal direction to the plan in order to generate a three dimensional shell mesh to perform the sequential formingcrashworthiness analysis for the beam. It is possible to perform both a radial and axial crashworthiness analysis. The user interface shown in Figure 6 allows the designer to specify the most crucial parameters for the simulation. It is easy to change the dimensions of the beam without redoing the whole 3Dmodel.

The next step is to generate the input files for the finite element solver and starts the analysis. When the analysis is finished the 
software scans the output files, created by the FE-solver, for the results. The results will then be presented for designer in a suitable way.

The solver used in this study is the implicit finite element code MSC.Marc2003. It was chosen because of the ease to make scripts and runs in batch mode. It is also possible to perform whole the sequential dynamic analysis regarding sheet metal forming, quasi-static analysis regarding the spring-back and finally dynamic analysis accounting for crash by the same code. However, only a few changes regarding the creation of the input files are needed to adapt the tool to work with another FE-solver.

These few steps enable the designer to have quick and relatively precise results for comparison between wider ranges of solutions and hence can work with a bigger solution space in the same time period it takes today to analyze one solution.

Scheme for demonstrator

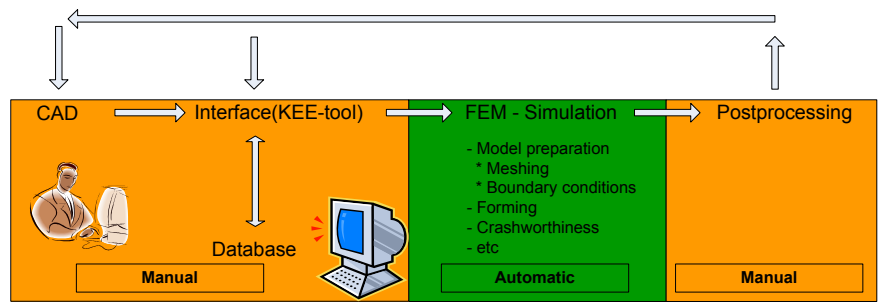

Figure 7. Scheme of KEE-application usage.

\subsection{The interface}

The actual application in this case is a simple to use graphical interface coded in Python, Figure 6. The decision to use Python is because of the flexibility to be able to run the script under different OS without need for recompilation of the code. The interface allows the designer to change the most crucial designand simulation parameters for the beam. The parameters and allowed values of these are stored as rules in the code and are based on ordinary physical laws, experience from senior analysts and company "rule-of-thumb" regarding analysis. A list of changeable parameters in the interface could be mentioned as in the following:

- material

- thickness of sheet

- length of extruded beam

- width of sheet to be formed in to designed beam

- velocity of forming

- velocity of crash

- choice of radial/axial crash simulation
The goal with the interface is that it must be easy to use and to understand for a designer with little experience in simulation.

\subsection{Creation of input files \& solving}

When the designer has entered the parameters in the interface the input files is created automatically. The demonstrator generates two files, one process file and one Python script. The process file starts MSC.Mentat2003 which is the pre-processor for Marc2003. The Python script is then started by the process file and executes the necessary commands to import the IGESfile, apply boundary conditions \& material, choice of element type, creation of load cases, and finally it generates the input file for the solver, 0 . The script imports the cross section, extrudes it the chosen length and then duplicates, with a scaling factor that's calculated from the chosen sheet thickness, it to get both. There are three load cases created, the first is a dynamic transient for the forming simulation, the second is a quasi-static load case for the spring back, and finally another dynamic transient for the crashworthiness analysis. All this is procedures takes approximately one minute and what the entire designer sees is Mentat2003 open, a set of commands being executed and then closes. When the demonstrator reckons the input file for the solver is ready it executes the solver and the simulation starts.

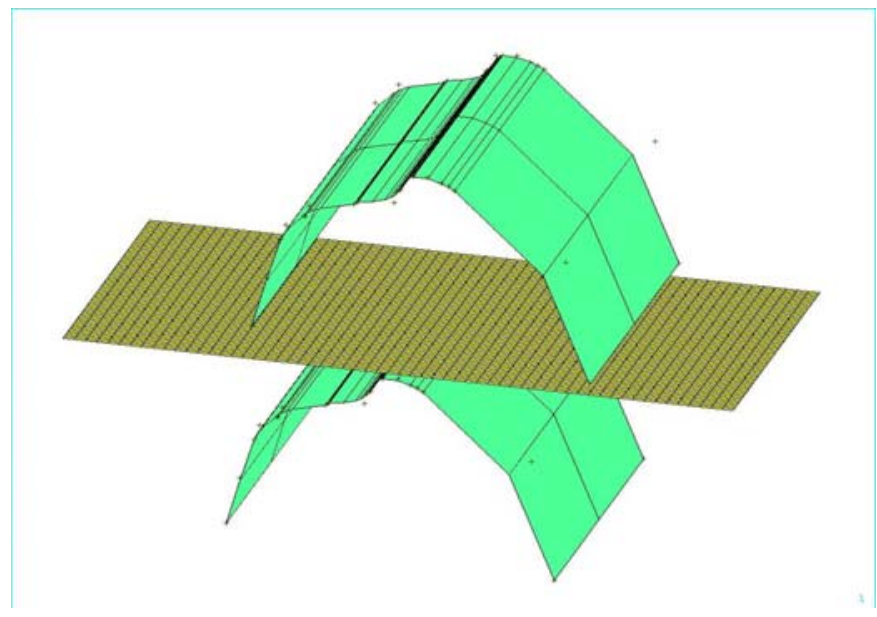

Figure 8. Example of geometry automatically created by the Python script.

\subsection{Results}

When the solver is finished the demonstrator scans the results files for selected results. Results are collected at two times. The first scan occurs after the forming simulation to check for sheet thinning and other crucial things that would make the real physical forming impossible. The second scan occurs when the complete simulation is finished. The results are then being 
presented for the designer in a suitable way with checks done against stored rules, Figure 9. Typical results:

- Thickness of element

- Maximum stress and strain

- The energy absorption

- Contact forces

The desired analysis time is less than twelve hours so the designer can start the simulation when he leaves the office and have the results ready by the next morning.

Example cross-section Axial load

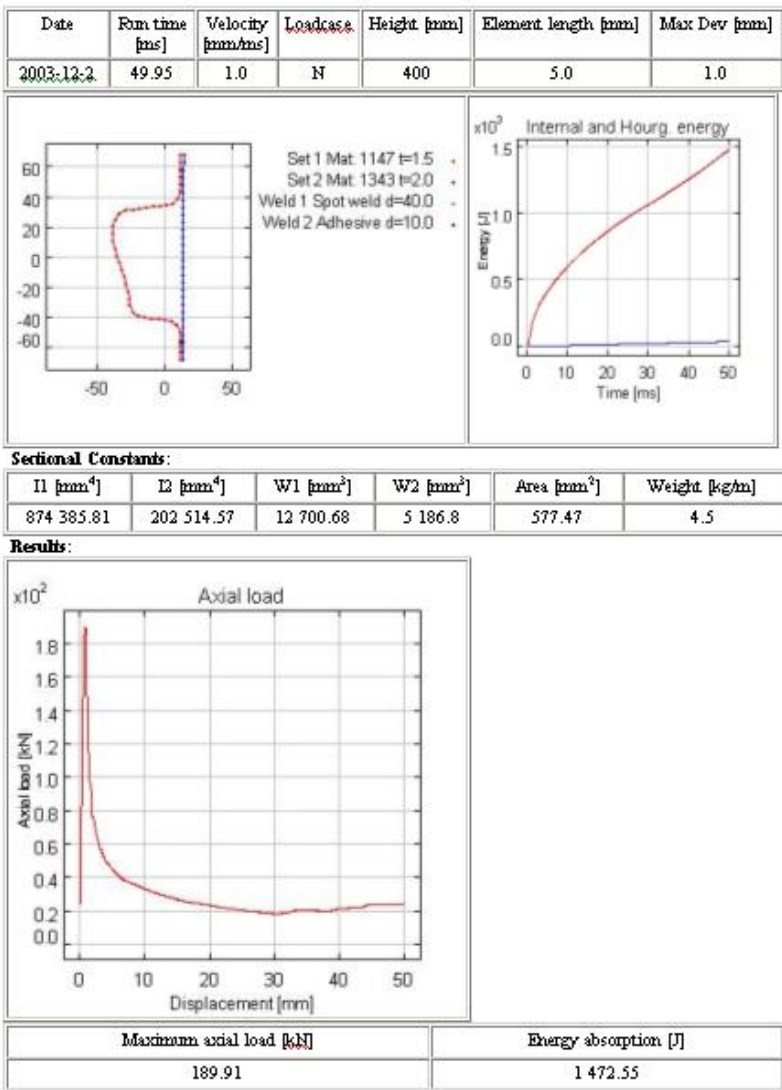

Figure 9. Result page from DAMIDA showing the amount of force and energy the beam has absorbed during the crashworthiness simulation. [23]

\section{DISCUSSION \& CONCLUSIONS}

Combining KEE methods and simulation technologies can improve the design evaluation process. Positive effects are the possibility of early standard analysis of design concepts; shorter analysis cycles (i.e. creating the possibility for optimization and more iteration) and the fact that experienced simulation experts can spend less time on routine tasks that are done by the KEE system users instead.
It is suggested that the Knowledge Enable Engineering (KEE) should be used as an integral part in engineering design process. On the other hand, the ED theory should define the boundary conditions for creating an efficient KEE system, Figure 10. It's also a useful support tool for FEM simulations, e.g., to handle the optimization loops.

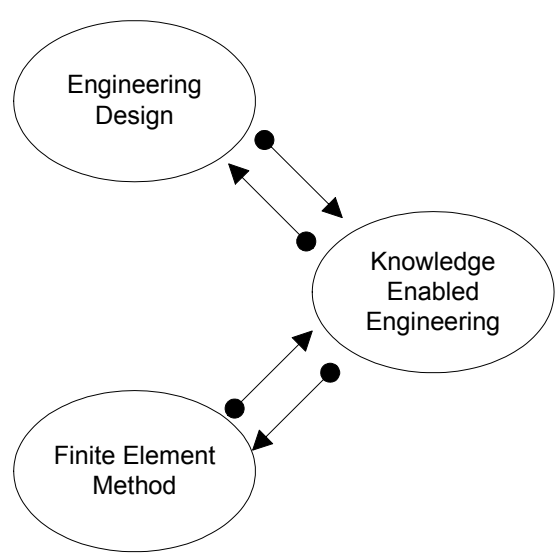

Figure 10. KEE is a useful tool both for a better coupling to FEM in the ED process and as a support tool for FEM simulations.

The demonstrator shows the ability to provide semi advanced analysis tools in a property based model (PBM) for a designer with little or non past experience in simulation. The benefit in the shortening of the design loops gives the designer the possibility to investigate a much higher number of solutions during the same period of time it took before. It also grants the designer the possibility to work with broken down requirements on a sub system level. As stated earlier this kind of applications will never replace the importance of a more advanced analyze made by a company senior analyst. It's more of guidance so the designer can make relative comparisons between different solutions to see if he is on the right track. This demonstrator also shows that there is no limit to this type of applications. This was only a first test of combining sheet metal forming and crashworthiness simulations but nothing prevents that more load cases are built in i.e. effects of heat treatment, local stresses from welding etc. It would also be possible to make optimization loops with the interface by just adding a few rows of code.

\section{ACKNOWLEDGMENTS}

This work has been founded by SSF (Swedish foundation for strategic research) Via the ProViking program which is acknowledged. The authors would also recognize the information made available by Volvo Car Corporation. 


\section{REFERENCES}

[1] Wang, L., Shen, W., Xie, H., Neelamkavil, J., Pardasani, A., 2002, "Collaborative conceptual design - state of the art and future trends" Computer-Aided Design, 34, pp. 981-996.

[2] Isakssson, O., 2003, "A generative modeling approach to engineering design" International Conference on Engineering Design, ICED’03, Stockholm, August 192.

[3] Hazelrigg G.A.., 1997, "On Irrationality in Engineering Design", Journal of Mechanical Design, vol. 119, Transactions of the ASME, pp.194-196.

[4] Barton, J., Love, D. M., et al, 2001, "Design determines $70 \%$ of cost? A review of implications for design evaluation" Journal of Engineering Design, 12, pp. 47-58.

[5] Phillips, R. E., 1997, "Dynamic objects for engineering automation" Communications of the ACM, 40, pp. 59-65.

[6] Pinfold, M., Chapman, C.,2001, "The Application of $\mathrm{KBE}$ techniques to the FE model creation of an automotive body structure", Computers in Industry. vol. 44, pp 1-10.

[7] Belytschko, T. and Hughers, T., Course literature (Paris, 2003).

[8] Mackerle, J., 2003, "International Journal of Machine Tools and Manufacture" 43 (1), 103-114.

[9] Marusich, T. and Ortiz, M., 1995, "International Journal of Numerical Methods in Engineering" 38, 3675-3694.

[10] Halil Bil , S. Engin Klç and A. Erman Tekkaya, "A comparison of orthogonal cutting data from experiments with three different finite element models" International Journal of Machine Tools and Manufacture, to appear.

[11] Hosford, W. and Duncan, J., 1999, "Sheet Metal Stamping, A Review" Journal of The Minerals, Metals \& Materials Society 51 (11), 39-44.

[12] Tekkaya, A. E.: 2001,"State-of-the-Art of Simulation of Sheet Metal Forming", Journal of Materials Processing Technology, 103, pp. 15-23.

[13] Gür, C.H.; Tekkaya, A. E., 2001, "Numerical investigation of non-homogeneous plastic deformation in quenching process", Materials Science and Engineering A, A319-321, pp. 164-169.
[14] Lindgren, L.-E., 2001, "Finite Element Modeling and Simulation of Welding part 1: Increased" Journal of Thermal Stresses 24, 141-192.

[15] Lindgren, L.-E., 2001, "Finite Element Modeling and Simulation of Welding part 2: Improved Material", Journal of Thermal Stresses 24, 195-231.

[16] Lindgren, L.-E., 2001, "FINITE ELEMENT MODELING AND SIMULATION OF WELDING. PART 3: EFFICIENCY AND INTEGRATION", Journal of Thermal Stresses 24, 305-334.

[17] Josefson, B., 1982, ASME Journal of Pressure Vessel Technology 104, 245-250.

[18] Berglund, D., Alberg, H., and Runnemalm, H., 2003, "Simulation of welding and stress relief heat treatment of an aero engine component", Finite Elements in Analysis and Design 39 (9), 865-88.

[19] Hyun, S. and Lindgren, L.-E., 2003, "Simulating a chain of manufacturing processes using a geometry based finite element code with adaptive meshing", Int. $\mathrm{J}$ Finite Elements in Analysis and Design to appear.

[20] Lindgren, L.-E., Runnemalm, H., Nguyen-Dang, H. et al., 1996, "Crack analysis of multi pass welded plate" in MMSP'96 General Workshop, Davos, Austria.

[21] Maddox, S., 1991, "Fatigue Strength of Welded Structures", Abington Publishing.

[22] Bylund, N., Isaksson,. Kalhori, V. \& Larsson, T. 2004, "Enhanced Engineering Design Practice Using Knowledge Enabled Engineering with Simulation Methods" In the proceedings of Design 2004 Conference, 18-20 of May 2004, Dubrovnik, Croatia

[23] Bylund, N. \& Eriksson, M., 2002, "Simulation Driven Car Body Development Using Property Based Models" SAE paper 2001-01-3046, in the proceedings of the International Body Engineering Conference, IBEC 2002, 8-12 of July 2002, Paris, France. 